Original article

\title{
PHYLOGENETIC ANALYSIS OF ESCHERICHIA COLI ISOLATES FROM HEALTHY AND DIARRHOEIC CALVES IN MASHHAD, IRAN
}

\author{
M. BARZAN ${ }^{1,2}$, M. RAD $^{1}$, G. R. HASHEMI TABAR ${ }^{1} \&$ M. AZIZZADEH ${ }^{3}$ \\ ${ }^{1}$ Department of Pathobiology, School of Veterinary Medicine, Ferdowsi University \\ of Mashhad, Mashhad, Iran; ${ }^{2}$ Department of Pathobiology, Shahid Chamran Univer- \\ sity of Ahvaz, Ahvaz, Iran; ${ }^{3}$ Department of Clinical Sciences, School of Veterinary \\ Medicine, Ferdowsi University of Mashhad, Mashhad, Iran
}

\section{Summary}

Barzan, M., M. Rad, G. R. Hashemi Tabar \& M. Azizzadeh, 2017. Phylogenetic analysis of Escherichia coli isolates from healthy and diarrhoeic calves in Mashhad, Iran. Bulg. J. Vet. Med., 20, No 1, 11-18.

Escherichia coli is a normal inhabitant of the gastrointestinal tract of vertebrates. Certain Escherichia coli strains have been associated with neonatal diarrhoea in ruminants. These strains can be assigned to one of the four main phylogenetic groups, A, B1, B2 and D. Several studies have shown the relationship between phylogeny and pathogenicity of $E$. coli, a great deal can be obtained by determining the phylogroup of unknown $E$. coli strains. In this study, we aimed to evaluate the influence of diarrhoea on the genetic composition of E. coli populations isolated from calves. A total of $80 \mathrm{ES}$ cherichia coli isolates were obtained from healthy and diarrhoeic calves. Phylogenetic grouping was done based on the Clermont triplex PCR method using primers targeted at three genetic markers, chuA, yjaA and TspE4.C2. According to our results, phylogenetic group A strains was the most prevalent in both healthy (37.5\%) and diarrhoeic calves (55\%). Group B1 contained $27.5 \%$ of isolates in healthy calves, followed by group B2 (17.5\%), and group D (7.5\%). Also, four isolates from healthy calves were not included in the major phylogenetic groups or subgroups. A total of $14 \%$ and $4 \%$ of isolates from diarrhoeic calves beloned to phylogroups B2 and D respectively. Although no isolate from diarrhoeic calves was found to belong to group B1, there was no significant difference between healthy and diarrhoeic calves for other phylogroups. There was not a dramatic shift in E. coli phylogroup/subgroup due to occurrence of diarrhoea in calves, except for phylogroup B1 which was higher in healthy calves. This can be due to the difference in secretions of digestive system in diarrhoeic calves which can prevent the conditions for instability of Escherichia coli isolates of phylogroup B1. The majority of isolates from both healthy and diarrhoeic calves belonged to nonpathogenic phylogentic group A and B1.

Key words: calves, diarrhoea, Escherichia coli, phylogenetic group 


\section{INTRODUCTION}

Escherichia coli is a normal inhabitant of the gastrointestinal tract of vertebrates. Its colonisation in the mammalian intestinal tract from environmental sources occurs shortly after birth and persists as one of the important members of the normal flora of the intestine throughout life (Quinn et al., 2011). Certain E. coli strains have been associated with neonatal diarrhoea in ruminants which causes considerable economic losses in dairy industry all around the world (Shahrani et al., 2014). E. coli strains may be assigned to one of the main phylogenetic groups: $\mathrm{A}, \mathrm{B} 1, \mathrm{~B} 2$ and $\mathrm{D}$, which classify into seven subgroups $\left(\mathrm{A}_{0}, \mathrm{~A} 1, \mathrm{~B} 1, \mathrm{~B} 2_{2}, \mathrm{~B} 2_{3}\right.$, $\mathrm{D}_{1}$ and $\left.\mathrm{D}_{2}\right)$, according to the combination of the two and three genetic markers (chuA, the outer-membrane hemin receptor gene, and yjaA, which encodes an uncharacterised protein) and a DNA fragment that has been recently identified as part of a putative lipase esterase gene, TspE4.C2 based on triplex PCR (Clermont et al., 2000; Escobar-Paramo et al., 2004; Tenaillon et al., 2010). This method, which assigns strains to their correct MLST-based phylogroup, is acceptably accurate (80-85\%) and has been found satisfactory (Gordon et al., 2008). Phylogenetic groups are different in characteristics such as virulence factors, ecologic niches, life history, carbohydrate fermentation, antibiotic resistance, growth rate and size of the genome (Bergthorsson et al., 1998; Lecointre et al., 1998; Walk et al., 2007).

Previous studies have shown that strains from phylogroups B2 and D contained more virulence factors than strains from the phylogroups A and B1 (Johnson et al., 2001; Bashir et al., 2012). The diarrhoeagenic E. coli strains belong to groups A, B1 and D, the commensal strains belong to groups $\mathrm{A}$ and $\mathrm{B} 1$, whilst the extra-intestinal pathogenic strains usually belong to groups B2 and D (Ferjani et al., 2012).

Up to now, there have been very few studies on phylogenetic group determination based on the health status of calves in Iran. Therefore, the aim of this study was to compare the phylogenetic groups of Escherichia coli isolates from healthy and diarrhoeic calves by Clermont triplex PCR method.

\section{MATERIALS AND METHODS}

\section{E. coli isolates}

This study was performed from September 2012 till July 2013, on 80 isolates of E. coli from faeces of 40 healthy calves and 40 calves with clinical diarrhoea. The bacterial strains were isolated from faecal samples of Holstein calves aged $<1$ month, from 5 farms located in northeast of Iran. The isolates were identified as $E$. coli based on standard biochemical tests (Seifi et al., 2015). Isolated strains which exhibited a biochemical profile for $E$. coli were kept as stock in nutrient broth with $15 \%$ glycerol at $-20{ }^{\circ} \mathrm{C}$ for further experiments.

\section{DNA extraction}

DNA template preparation was performed by the boiling method as followed. First, a few colonies were resuspended in $500 \mu \mathrm{L}$ sterile distilled water. The cells were lysed by heating at $95{ }^{\circ} \mathrm{C}$ for $10 \mathrm{~min}$. After heating, they were immediately put on ice for $5 \mathrm{~min}$. The supernatant was then harvested by centrifugation at $11,000 \mathrm{rpm}$ for $10 \mathrm{~min}$. 
Determination of E. coli phylogenetic groups

We determined four phylogenetic groups of $E$. coli (A, B1, B2 and D) by use of triplex PCR as described by Clermont et al. (2000). Briefly, the genomic DNA of bacterial strains was amplified by triplex PCR using primers targeted at three markers, chuA, yjaA and TspE4.C2. The primer pairs used for PCR amplification is shown in Table 1. Multiplex PCR reaction was performed in a $25 \mu \mathrm{L}$ reaction mixture, containing PCR buffer $(10 \mathrm{mM}$ Tris- $\mathrm{HCl}, 50 \mathrm{mM} \mathrm{KCl}$, and $1.5 \mathrm{mM}$ $\mathrm{MgCl}_{2}, \mathrm{pH}$ 8.7), dNTP $(200 \mu \mathrm{M})$, each primer $(0.4 \mu \mathrm{M})$, Taq DNA polymerase (1U), and template DNA $(2 \mu L)$. Negative controls (reaction lacking the template DNA) and a positive control (ECOR 62) were included in all performed amplifications. The PCR reaction was performed as follows: initial denaturation at $94{ }^{\circ} \mathrm{C}$ for $5 \mathrm{~min}, 30$ cycles of 30 $\mathrm{s}$ at $94{ }^{\circ} \mathrm{C}, 30 \mathrm{~s}$ at $55{ }^{\circ} \mathrm{C}$ and $30 \mathrm{~s}$ at 72 ${ }^{\circ} \mathrm{C}$, followed by a final extension step at $72{ }^{\circ} \mathrm{C}$ for $7 \mathrm{~min}$ (Clermont et al., 2000). Reactions were placed in a thermal cycler (Biorad, Germany) without mineral oil. Amplification products were separated in $1.5 \%$ agarose gels containing ethidium bromide.

\section{Statistical analysis}

Phylogenetic relationship between the groups and subgroups and health status of calves (diarrhoeic and healthy) was evaluated by SPSS 20 using the Chisquare and Fisher exact tests with significance set at $\mathrm{P}<0.05$.

\section{RESULTS}

Subtype distribution of isolates is shown in Table 2. According to multiplex PCRbased phylotyping, group A contained the majority of the collected isolates from both healthy and diarrhoeic calves. A total of 15 isolates $(37.5 \%)$ from healthy calves belonged to phylogenetic group A, followed by group B2 (11 isolates, $27.5 \%)$, B1 (7 isolates, $17.5 \%)$, and D (3 isolates, $7.5 \%)$. All of the strains of group A were found to belong to subgroup $A_{1}$. Four and seven isolates $(10 \%$ and $17.5 \%$ ) of group B2 belonged to subgroup $\mathrm{B} 22_{2}$ and $\mathrm{B} 23_{3}$, respectively. $\mathrm{A}$ total of 22 isolates $(55 \%)$ from diarrhoeic calves belonged to group A, followed by group B2 (14 isolates, 35\%), and group D (4 isolates, 10\%). No strains were found to belong to group B1. All strains of group A were found to belong to subgroup $A_{1}$. Nine and five isolates $(22.5 \%$ and $12.5 \%$ ) of group B2 belonged to

Table 1. Oligonucleotid primers used for detection of phylogenetic groups

\begin{tabular}{|c|c|c|c|}
\hline Primer name & Sequence $\left(5^{\prime}-3^{\prime}\right)$ & $\begin{array}{l}\text { Product size } \\
\text { (bp) }\end{array}$ & Target gene \\
\hline $\begin{array}{l}\text { ChuA.1 } \\
\text { ChuA.2 }\end{array}$ & $\begin{array}{l}\text { GAC GAA CCA ACG GTC AGG AT } \\
\text { TGC CGC CAG TAC CAA AGA CA }\end{array}$ & $279 \mathrm{bp}$ & chuA \\
\hline $\begin{array}{l}\text { YjaA.1 } \\
\text { YjaA.2 }\end{array}$ & $\begin{array}{l}\text { TGAAGTGTCAGGAGACGCTG } \\
\text { ATG GAG AAT GCG TTC CTC AAC }\end{array}$ & $211 b p$ & yjaA \\
\hline $\begin{array}{l}\text { TspE4.C2.1 } \\
\text { TspE4.C2.2 }\end{array}$ & $\begin{array}{l}\text { GAG TAA TGT CGG GGC ATT CA } \\
\text { CGC GCC AAC AAA GTA TTA CG }\end{array}$ & $152 \mathrm{bp}$ & TspE4.C2 \\
\hline
\end{tabular}


Phylogenetic analysis of E. coli isolates from healthy and diarrhoeic calves in Mashhad, Iran

Table 2. Phylogenetic analysis of 80 E. coli isolates

\begin{tabular}{lccc}
\hline & \multicolumn{3}{c}{ Number (\%) of isolates } \\
\cline { 2 - 4 } Phylogroup/subgroup & $\begin{array}{c}\text { from healthy } \\
\text { calves }\end{array}$ & $\begin{array}{c}\text { from diarrhoeic } \\
\text { calves }\end{array}$ & $\begin{array}{c}\text { from all } \\
\text { calves }\end{array}$ \\
\hline $\mathrm{A}_{0}$ (chuA-, yjaA-, TspE4.C2-) & - & - & - \\
$\mathrm{A}_{1}($ chuA-, yjaA+, TspE4.C2-) & $15(37.5)$ & $22(55.0)$ & $37(46.25)$ \\
$\mathrm{B} 1$ (chuA-, yjaA-, TspE4.C2+) & $7(17.5)$ & - & $7(8.75)$ \\
$\mathrm{B} 2_{2}($ chuA+, yjaA+, TspE4.C2-) & $4(10.0)$ & $9(22.5)$ & $13(16.25)$ \\
$\mathrm{B}_{3}(\mathrm{chuA}+$, yjaA+, TspE4.C2+) & $7(17.5)$ & $5(12.5)$ & $12(15.0)$ \\
$\mathrm{D}_{1}(\mathrm{chuA}+$, yjaA-, TspE4.C2-) & $1(2.5)$ & $4(10.0)$ & $5(6.25)$ \\
$\mathrm{D}_{2}($ chuA+, yjaA-, TspE4.C2+) & $2(5.0)$ & - & $2(2.5)$ \\
Untypable (chuA-, yjaA+, TspE4.C2+) & $4(10.0)$ & - & $4(5.0)$ \\
\hline Total & 40 & 40 & 80 \\
\hline
\end{tabular}

subgroup $\mathrm{B} 2_{2}$ and $\mathrm{B} 2_{3}$, respectively. All strains of group D were found to belong to subgroup $\mathrm{D}_{1}$. No strains from healthy and diarrhoeic calves were found to belong to subgroup $\mathrm{A}_{0}$. Four isolates were untypeable by this method (Fig. 1).

There was not any significant difference between healthy and diarrhoeic calves for $E$. coli phylogroups distribution $(\mathrm{P}=0.217)$. The binary comparison of phylogenetic group B1 showed statistically significantly higher frequency of group B1 among isolates of healthy than among isolates of diarrhoeic calves $(\mathrm{P}=0.012)$.

\section{DISCUSSION}

Previous studies have shown that phylogenetic groups, subgroups and genetic markers are not randomly distributed in different hosts such as human and animals and that the frequency of phylogenetic groups in mammals is dependent on host food regimen, body volume and climate conditions (Gordon \& Cowling, 2003; Mokracka et al., 2011). In this study, 80 Escherichia coli strains isolated from faecal samples of healthy and diarrhoeic calves, were evaluated. There was not any significant difference between healthy and diarrhoeic calves for $E$. coli phylogroups distribution $(\mathrm{P}=0.217)$, but the binary comparison of phylogenetic group B1 showed that the frequency of group B1 in isolates from healthy calves was higher than that of isolates from diarrhoeic calves $(\mathrm{P}=0.012)$. All phylogenetic groups and subgroups were present in isolates of healthy and diarrhoeic calves, but no isolates were found to belong to subgroup $A_{0}$. Subgroup $D_{2}$ and phylogroup B1 were not found among isolates from diarrhoeic calves. This can be due to the difference in secretions of digestive system in diarrhoeic calves which can prevent the conditions for instability of Escherichia coli isolates. E. coli strains in gut normal microflora may therefore be the natural reservoir of pathogenic strains and they may be derived from commensal strains by the acquisition of chromosomal or extrachromosomal virulence operons (Duriez 


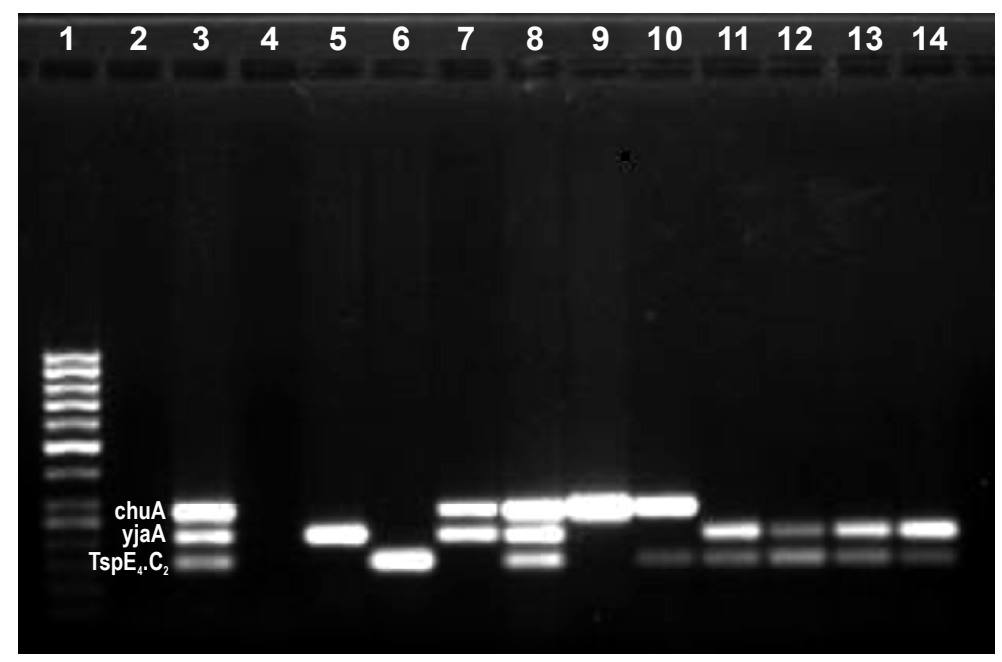

Fig. 1. Triplex PCR profiles specific for E. coli phylogenetic groups. Each combination of $c h u A$ and yjaA genes and DNA fragment TSPE4.C2 amplification allowed phylogenetic group determination of a strain. Lane 1: Marker 100 bp plus (Fermentas); lanes 2 and 4: negative controls; lane 3: positive control; lane 5: Group A (A1), lane 6: Group B1, lanes 7-8: Group B2; lanes 9-10: Group D and lanes 11-14: untypeable.

et al., 2001). In our study the majority of isolates belonged to the non-pathogenic phylogenetic group A, but certain virulence factors may be mobilised on genetic elements and transferred to normally commensal strains via horizontal exchange (Picard \& Goullet, 1988). Our data showed that phylogoup $\mathrm{A}\left(\mathrm{A}_{1}\right)$ was the most prevalent among isolates from healthy and diarrhoeic calves. This result was similar to previous studies which showed that isolates of phylogroup A were the most prevalent $E$. coli isolates in animal intestinal tract (Johnson et al., 2003; Escobar-Paramo et al., 2006; Asai et al., 2011). Other studies revealed that phylogroup B1 was dominant among isolates from healthy cattle (Alizade et al., 2014). The analysis demonstrated that phylogroup B1 was more prevalent in isolates from diarrhoeic and septicemic calves in Iran (Ghanbarpour et al., 2009; Bihannic et al., 2014; Staji et al.,
2015). It is interesting to note that subgroup $\mathrm{B} 22_{3}$ was found among isolates from both healthy and diarrhoeic calves, whereas subgroup $\mathrm{A}_{0}$ was not found in all isolates. These results are in contrast with the study conducted by Carlos et al. (2010) - according to their results subgroup B2 3 was present only in the human sample and they suggested that B2 strains, especially subgroup $\mathrm{B} 2_{3}$, could be a good indicator of human faeces contamination. It was similar to previous study which showed that isolates of ruminants (sheep, goat and cattle) and dogs belonged to subgroup B2 3 (Derakhshandeh et al., 2014). It is difficult to explain but environmental and ecological conditions, geographic variation, host species and health status play an important role in E. coli phylogroup distribution.

In conclusion, our results showed that there was not a significant shift in $E$. coli phylogroup/subgroup due to occurrence 
of diarrhoea. Its effect on the presence or absence of some phylo-groups was obvious. As different phylogroups have various features in many aspects such as ability to cause disease, these findings would be important to formulate prevention programmes and effective therapies for calves' diseases. Further studies need to be done on the shift of E. coli phylogroups related to the health status of calves in different geographical regions of Iran.

\section{ACKNOWLEDGMENTS}

This project was supported by research grant (grant no 3/25217) of Ferdowsi University of Mashhad. The authors wish to thank Dr Ghanbarpour for kindly providing the strain ECOR 62, and Mrs. Gholamhosseini Moghadam for her technical assistance.

\section{REFERENCES}

Alizade, H., R. Ghanbarpour \& M. Nekoubin, 2014. Phylogenetic of Shiga toxin-producing Escherichia coli and a typical enteropathogenic Escherichia coli strains isolated from human and cattle in Kerman, Iran. International Journal of Enteric Pathogens, 2, e15195.

Asai, K. M. T., Ch. Sato, M. Hiki, M. Usui, K. Baba, M. Ozawa, K. Harada, H. Aoki \& T. Sawada, 2011. Phylogenetic groups and cephalosporin resistance genes of EScherichia coli from diseased foodproducing animals in Japan. Acta Veterinaria Scandinavica, 53, 52.

Bashir, S., A. Haque, Y. Sarwar, A. Ali \& M. Irfan Anwar, 2012. Virulence profile of different phylogenetic groups of locally isolated community acquired uropathogenic E. coli from Faisalabad region of Pakistan. Annals of Clinical Microbiology and Antimicrobials, 11, 23.

Bergthorsson, U. \& H. Ochman, 1998. Distribution of chromosome length variation in natural isolates of Escherichia coli. Molecular Biology and Evolution, 15, 616.

Bihannic, M., R. Ghanbarpour, F. Auvray, L. Cavalié, P. Châtre, M. Boury, H. Brugère, J. Y. Madec \& E. Oswald, 2014. Identification and detection of three new F17 fimbrial variants in Escherichia coli strains isolated from cattle. Veterinary Research, 45, 76.

Carlos, C., M. M. Pires, N. C. Stoppe, E. M. Hachich, M. I. Z. Sato, T. A. T. Gomes, L. A. Amaral \& L. M. M. Ottoboni, 2010. Escherichia coli phylogenetic group determination and its application in the identification of the major animal source of fecal contamination. BMC Microbiology, 10, 161.

Clermont, O., S. Bonacorsi \& E. Bingen, 2000. Rapid and simple determination of the Escherichia coli phylogenetic group. Applied and Environmental Microbiology, 66, 4555-4558.

Derakhshandeh, A., R. Firouzi \& Z. Naziri, 2014. Phylogenetic group determination of faecal Escherichia coli and comparative analysis among different hosts. Iranian Journal of Veterinary Research, 15 , 13-17.

Duriez, P., O. Clermont, S. Bonacorsi, E. Bingen, A. Chaventre, J. Elion, B. Picard \& E. Denamur, 2001. Commensal Escherichia coli isolates are phylogenetically distributed among geographically distinct human populations. Microbiology, 147, 1671-1676.

Escobar-Páramo, P., A. Le Menac'h, T. Le Gall, C. Amorin, S. Gouriou, B. Picard, D. Skurnik \& E. Denamur, 2006. Identification of forces shaping the commensal Escherichia coli genetic structure by comparing animal and human isolates. Environmental Microbiology, 8, 1975 1984.

Escobar-Paramo, P., K. Grenet, A. Le Menac'h, L. Rode, E. Salgado, C. Amorin, S. Gouriou, B. Picard, M. C. Rahimy, A. Andremont, E. Denamur \& R. Rui- 
my, 2004. Large-scale population structure of human commensal Escherichia coli isolates. Applied and Environmental Microbiology, 70, 5698-5700.

Ferjani, S., M. Saidani, S. Ennigrou, M. Hsairi \& S. Ben Redjeb, 2012. Virulence determinants, phylogenetic groups and fluoroquinolone resistance in Escherichia coli isolated from cystitis and pyelonephritis. Pathologie- Biologie (Paris), 60, 270-274.

Ghanbarpour, R. \& E. Oswald, 2009. Characteristics and virulence genes of Escherichia coli isolated from septicemic calves in southeast of Iran. Tropical Animal Health and Production, 41, 10911099.

Ghanbarpour, R. \& E. Oswald, 2010. Phylogenetic distribution of virulence genes in Escherichia coli isolated from bovine mastitis in Iran. Research in Veterinary Science, 88, 6-10.

Gordon, D. M. \& A. Cowling, 2003. The distribution and genetic structure of Escherichia coli in Australian vertebrates: host and geographic effects. Microbiology, 149, 3575-3586.

Gordon, D. M., O. Clermont, H. Tolley \& E. Denamur, 2008. Assigning Escherichia coli strains to phylogenetic groups: multilocus sequence typing versus the PCR triplex method. Environmental Microbiology, 10, 2484-2496.

Johnson, J. R., P. Delavari, M. Kuskowski \& A. L. Stell, 2001. Phylogenetic distribution of extraintestinal virulence-associated traits in Escherichia coli. Journal of Infectious Disease, 183, 78-88.

Johnson, J. R., M. A. Kuskowski, K. Owens, A. Gajewski \& P. L. Winokur, 2003. Phylogenetic origin and virulence genotype in relation to resistance to fluoroquinolones and/or extended-spectrum cephalosporins and cephamycins among Escherichia coli isolates from animals and humans. Journal of Infectious Disease, 188, 759-768.
Lecointre, G., L. Rachdi, P. Darlu \& E. Denamur, 1998. Escherichia coli molecular phylogeny using the incongruence length difference test. Molecular Biology and Evolution, 15, 1685-1695.

Mokracka, J., L. Jablonska, R. Koczura \& A. Kaznowski, 2011. Phylogenetic groups, virulence genes and quinolone resistance of integron-bearing Escherichia coli strains isolated from a wastewater treatment plant. Antonie van Leeuwenhoek, 99, 817-824

Picard, B. \& P. Goullet, 1988. Correlation between electrophoretic types $\mathrm{B}_{1}$ and $\mathrm{B}_{2}$ of carboxylesterase B and host-dependent factors in Escherichia coli septicaemia. Epidemiology and Infection, 100, 51-61.

Quinn, P. J., B. K. Markey, F. C. Leonard, E. S. Fitz Patrick, S. Fanning \& P. J. Hortigan, 2011. Veterinary microbiology and microbial disease. In: Veterinary Microbiology, $2^{\text {nd }}$ edn, Wiley-Blackwell, UK, p. 309.

Seifi, S., R. Khoshbakht \& A. R. Atabak, 2015. Antibiotic susceptibility, serotyping and pathogenicity evaluation of avian EScherichia coli isolated from broilers in northern Iran. Bulgarian Journal of Veterinary Medicine, 18, 173-179.

Shahrani, M., F. Safarpoor Dehkordi \& H. Momtaz, 2014. Characterization of Escherichia coli virulence genes, pathotypes and antibiotic resistance properties in diarrheic calves in Iran. Biological Research, 47, 28

Staji, H., A. Tonelli, A. Javaheri-Vayeghan, E. Changizi \& MR. Salimi-Bejestani, 2015. Distribution of Shiga toxin genes subtypes in B1 phylotypes of Escherichia coli isolated from calves suffering from diarrhea in Tehran suburb using DNA oligonucleotide arrays. Iranian Journal of Microbiology, 7, 191-197.

Tenaillon, O., D. Skurnik, B. Picard \& E. Denamur, 2010. The population genetics of commensal Escherichia coli. Nature Reviews Microbiology, 8, 207-217. 
Phylogenetic analysis of E. coli isolates from healthy and diarrhoeic calves in Mashhad, Iran

Walk, S. T., E. W. Alm, L. M. Calhoun, J. M. Mladonicky \& T. S. Whittam, 2007. Genetic diversity and population structure of Escherichia coli isolated from freshwater beaches. Environmental Microbiology, 9, 2274-2288.

Paper received 17.08.2015; accepted for publication 12.11.2015

\section{Correspondence:}

Mehrnaz Rad

Department of Pathobiology,

School of Veterinary Medicine,

Ferdowsi University of Mashhad,

P.O.Box: 91775-1793

Mashhad, Iran

e-mail:rad@um.ac.ir

mehrnazrad@yahoo.com 